PROCEEDINGS OF THE

AMERICAN MATHEMATICAL SOCIETY

Volume 128, Number 12, Pages 3655-3660

S 0002-9939(00)05805-6

Article electronically published on June 7, 2000

\title{
ESTIMATES IN SURFACES WITH POSITIVE CONSTANT GAUSS CURVATURE
}

\author{
JOSÉ A. GÁLVEZ AND ANTONIO MARTÍNEZ \\ (Communicated by Christopher Croke)
}

\begin{abstract}
We give optimal bounds of the height, curvature, area and volume of $K$-surfaces in $\mathbb{R}^{3}$ bounding a planar curve. The spherical caps are characterized as the unique $K$-surfaces achieving these bounds.
\end{abstract}

\section{INTRODUCTION}

One of the main geometric concepts in two-dimensional Riemannian geometry is the Gaussian curvature - it has been playing an essential role in surface theory. In fact, compact smooth surfaces immersed in $\mathbb{R}^{3}$ with constant positive Gauss curvature $K, K$-surfaces, form a class which has been receiving great interest.

Since a closed $K$-surface (that is, without boundary) is itself a round sphere, the surfaces in which we are interested have non-empty boundary. In this spirit some natural problems that arise are the problems of existence, uniqueness, structure of the space of $K$-surfaces bounding a given boundary and the properties about the geometry and topology of $K$-surfaces. Most of these problems seem far from being understood. We mention some results proved by Cafferelli, Guan, Nirenberg, Rosenberg and Spruck about the existence of $K$-graphs (see [2], [4] and [5]).

In this paper we consider $S$ a compact surface with a connected boundary, $\partial S$, and $x: S \longrightarrow \mathbb{R}^{3}$ a $K$-surface such that $\Gamma=x(\partial S)$ is a curve in a plane $P$.

If $\Gamma$ is a Jordan curve, Hoffman, Rosenberg and Spruck proved (by using elementary facts, see [5]) that $\Gamma$ is free of inflexion points, $P$ meets $x(S)$ transversally and $x(S)$ is fibred by strictly Jordan curves in parallel planes to $P$ (in particular, $x$ is an embedding and $x(S) \cup \Omega$ is the boundary of a convex body, where $\Omega$ is a strictly convex domain in $P$ with boundary $\Gamma$ ). Our objective in this paper is to study properties of the immersion $x$ related to non-existence problems and behaviour of its area and volume. The main results are obtained by proving optimal estimates of the height, curvature, area and enclosed volume that the immersion must satisfy.

If $x$ is an embedding, H. Rosenberg proved the following inequality in [6]:

$$
h \sqrt{K} \leq 2,
$$

where by $h$ we will denote the maximum height that $x(S)$ can rise above the plane containing $\Gamma$. We include a different proof of (11) to make this paper self-contained.

Received by the editors February 24, 1999.

2000 Mathematics Subject Classification. Primary 53A05.

Key words and phrases. K-surfaces, height, area, volume.

This research was partially supported by DGICYT Grant No. PB97-0785.

(C)2000 American Mathematical Society 
Among the main results we prove, are the following:

Theorem 1. Let $\bar{A}$ be the algebraic area of $\Gamma$. Then

$$
K \leq \frac{\pi|i(\Gamma)|}{|\bar{A}|}
$$

where $i(\Gamma)$ is the rotation index of $\Gamma$.

Moreover, if the Euler-Poincaré characteristic of $S, \chi(S)$, is 1, then the equality holds if and only if $x(S)$ is a hemisphere of radius $1 / \sqrt{K}$.

Theorem 2. Let $A$ be the area of $x(S)$. Then

$2 \pi \chi(S)-2 \sqrt{\pi^{2} i(\Gamma)^{2}-\pi K|\bar{A}||i(\Gamma)|} \leq K A \leq 2 \pi \chi(S)+2 \sqrt{\pi^{2} i(\Gamma)^{2}-\pi K|\bar{A}||i(\Gamma)|}$.

Moreover, if $\chi(S)=1$, then the equality holds in some of the above inequalities if and only if $x(S)$ is a spherical cap.

Theorem 3. If $x(S)$ is a graph on a domain in the plane $P$, then the enclosed volume by $x(S)$ and $P$ must satisfy the following inequality:

$$
V \leq \pi\left(\frac{h}{K}-\frac{h^{3}}{3}\right)
$$

where $h$ is the maximum height of $x(S)$ to $P$. Moreover, the equality holds if and only if $x(S)$ is a hemisphere of radius $1 / \sqrt{K}$.

\section{Height and Curvature estimates}

Let $P=\left\{v \in \mathbb{R}^{3} \mid\langle v, a\rangle=0\right\}$ be the plane containing $\Gamma$, where $a$ is a unit vector. Since $K$ is positive, $S$ is orientable and we can choose a unit normal to the immersion, $N$, such that the metric associated with the second fundamental form, $\sigma$, is positive definite. Then we have

Lemma 1. If $x$ is an embedding, then the maximum height that $x(S)$ can rise above $P$ is $2 / \sqrt{K}$.

Proof. In [3] it is proved

$$
\begin{gathered}
\Delta^{\sigma} x=2 N, \\
\Delta^{\sigma} N=-2 H N,
\end{gathered}
$$

where $\Delta^{\sigma}$ is the Laplacian for the metric, $\sigma$, given by the second fundamental form and $H$ is the mean curvature of the immersion.

Let us assume $x(S)$ is a graph on $P$ into $P^{+}=\left\{v \in \mathbb{R}^{3} \mid\langle v, a\rangle \geq 0\right\}$. Then the height function to $P$ satisfies

$$
\Delta^{\sigma}\langle\sqrt{K} x+N, a\rangle=2(\sqrt{K}-H)\langle N, a\rangle \geq 0 \text { on } S,
$$

and

$$
\langle\sqrt{K} x+N, a\rangle=\langle N, a\rangle \leq 0 \quad \text { on } \partial S .
$$

Thus, $\langle\sqrt{K} x+N, a\rangle \leq 0$ on $S$, that is,

$$
\langle x, a\rangle \leq-\frac{1}{\sqrt{K}}\langle N, a\rangle \leq \frac{1}{\sqrt{K}} .
$$

In the general case, $x(S)$ is in a side of the plane $P$ (see, for instance, [5]) and the proof follows, in a standard way, from the maximum principle by using Alexandroff reflection with parallel planes to $P$ coming down from the higher point. 
Lemma 2. If $\chi(S)=1$ and $\Gamma$ is a line of curvature (not planar necessarily), then $x(S)$ is contained in a sphere of radius $1 / \sqrt{K}$.

Proof. Let us consider $S$ as a Riemann surface with the conformal structure given by its second fundamental form, $\sigma$. Since $\chi(S)=1$, then $S$ is conformally equivalent to the closed unit disk $D=\{z \in \mathbb{C}|| z \mid \leq 1\}$.

Thus, we can consider $S=D$ and choose polar coordinates $(r, \theta)$ given by $z=u+i v=r e^{i \theta}$. Therefore,

$$
\begin{gathered}
\frac{\partial}{\partial r}=\cos \theta \frac{\partial}{\partial u}+\sin \theta \frac{\partial}{\partial v} \\
\frac{\partial}{\partial \theta}=-\sin \theta \frac{\partial}{\partial u}+\cos \theta \frac{\partial}{\partial v}
\end{gathered}
$$

on $\partial D$. Assuming that $(u, v)$ are conformal parameters we have

$$
\sigma\left(\frac{\partial x}{\partial r}, \frac{\partial x}{\partial \theta}\right)=0
$$

From the above equation and because $\Gamma$ is a line of curvature, we obtain

$$
0=\left\langle\frac{\partial x}{\partial r}, \frac{\partial x}{\partial \theta}\right\rangle=-\frac{1}{2} \sin 2 \theta(E-G)+\cos 2 \theta F \quad \text { on } \partial D,
$$

where

$$
E=\left\langle\frac{\partial x}{\partial u}, \frac{\partial x}{\partial u}\right\rangle, \quad F=\left\langle\frac{\partial x}{\partial u}, \frac{\partial x}{\partial v}\right\rangle \quad \text { and } \quad G=\left\langle\frac{\partial x}{\partial v}, \frac{\partial x}{\partial v}\right\rangle .
$$

On the other hand, $f(z)=E(z)-G(z)-2 i F(z)$ is a holomorphic function (see [3]) and so is $g(z)=z^{2} f(z)$. But the imaginary part of $g$ is a harmonic function on $D$ and it is given by $\sin 2 \theta(E-G)-2 \cos 2 \theta F$ on $\partial D$. Then, from (3) the imaginary part of $g$ vanishes and $g$ must be constant.

As $g(0)=0$ then $f=0$. That is, $x$ is a totally umbilical immersion and, therefore, $x(S)$ is contained in a sphere of radius $1 / \sqrt{K}$.

Proof of Theorem 1. Let $(u, v)$ be local coordinates of the immersion. We have

$$
N_{u} \wedge N_{v}=K x_{u} \wedge x_{v}
$$

that is,

$$
K(d(d x \wedge x))=d(d N \wedge N) .
$$

By applying Stoker's theorem, we have the following balancing formula:

$$
K \int_{\Gamma} d x \wedge x=\int_{\Gamma} d N \wedge N .
$$

As $x$ is a $K$-surface, $P$ must meet $\Gamma$ transversally and $N \wedge a \neq 0$ along its boundary. We can choose a well-oriented unit tangent vector field $t$ along $\Gamma$ such that $\operatorname{det}(N, t, a)>0$ on $\Gamma$. Then, from (44),

$$
\begin{aligned}
2 K|\bar{A}| & =K \int_{\Gamma}\langle d x \wedge x, a\rangle=\int_{\Gamma}\langle d N \wedge N, a\rangle \\
& =\int_{\Gamma} k_{n} \operatorname{det}(N, t, a) \leq \int_{\Gamma} k_{\Gamma}=2 \pi|i(\Gamma)|,
\end{aligned}
$$

where

$$
\bar{A}=\frac{1}{2} \int_{\Gamma}\langle x \wedge d x, a\rangle
$$


is the algebraic area of $\Gamma$ and $k_{n}, k_{\Gamma}$ are the normal curvature and curvature of $\Gamma$, respectively.

If the equality holds in the above inequality, then $N \wedge t=a$ and $k_{n}=k_{\Gamma}$ along Г. Thus,

$$
\begin{aligned}
\langle N, a\rangle & =0 \\
\langle d N(t), N \wedge t\rangle & =\langle d N(t), a\rangle=0
\end{aligned}
$$

and

$$
d N(t)=-k_{\Gamma} t .
$$

Therefore, $x(\partial S)$ is a line of curvature. Moreover, if $\chi(S)=1$, then, from Lemma $2, x(S)$ is contained in a sphere of radius $1 / \sqrt{K}$.

Remark 1. The algebraic area of $\Gamma$ only depends on the curve $\Gamma$ and not on its representation $\left.x\right|_{\partial S}$. Moreover, if $\Gamma$ is a Jordan curve, then $|\bar{A}|$ is the area of the interior of $\Gamma$ and $|i(\Gamma)|=1$.

Corollary 1. If $\Gamma$ is a planar Jordan curve, then

$$
K \leq \frac{\pi}{|\bar{A}|}
$$

and the equality holds if and only if $x(S)$ is a hemisphere of radius $1 / \sqrt{K}$.

Proof. It follows from Theorem 1 because if $\Gamma$ is a Jordan curve, then $x$ is an embedding and $\chi(S)=1$.

\section{Area and volume estimates}

Proof of Theorem 2. We can choose a well-oriented unit tangent vector field $t$ on $\Gamma$, such that $\operatorname{det}(N, t, a)<0$ and the covariant derivative of $t$ along $\Gamma, t^{\prime}$ is written as

$$
t^{\prime}=k_{g}(N \wedge t)+k_{n} N,
$$

where $k_{g}$ and $k_{n}$ are the geodesic and normal curvature of $\Gamma$, respectively.

We can assume a neighborhood of $\Gamma$ in $x(S)$ is on $P^{+}=\left\{y \in \mathbb{R}^{3} \mid\langle y, a\rangle \geq 0\right\}$. Thus,

$$
\left\langle t^{\prime}, a\right\rangle=-k_{g}|\nabla f|+k_{n}\langle N, a\rangle,
$$

where $f=\langle x, a\rangle$ is the height function and $\nabla f$ is its gradient in the induced metric on $S$.

On the other hand, if $n$ is the inner unit normal to $\Gamma$ in $P$, we have

$$
N=\langle N, n\rangle n+\langle N, a\rangle a=|\nabla f| n+\langle N, a\rangle a .
$$

Consequently,

$$
|\nabla f|^{2}+\langle N, a\rangle^{2}=1, \quad k_{g}^{2}=k_{\Gamma}^{2}\left(1-|\nabla f|^{2}\right) .
$$


Now, by using the Gauss-Bonnet Theorem, the Schwarz inequality, (5) and (7) we obtain,

$$
\begin{aligned}
(2 \pi \chi(S)-K A)^{2} & =\left(\int_{\Gamma} k_{g}\right)^{2} \leq \int_{\Gamma} k_{\Gamma} \int_{\Gamma} k_{\Gamma}\left(1-|\nabla f|^{2}\right) \\
& =\int_{\Gamma} k_{\Gamma}\left(\int_{\Gamma} k_{\Gamma}-\int_{\Gamma} k_{n} \operatorname{det}(N, t,-a)\right) \\
& =(2 \pi|i(\Gamma)|)(2 \pi|i(\Gamma)|-2 K \bar{A}),
\end{aligned}
$$

which gives the inequalities in the theorem.

Moreover, if the equality holds in (8) , then $|\nabla f|$ must be constant along $\Gamma$ and consequently $\langle N, a\rangle$ is constant on $\Gamma$. Since $d N(t)=k_{n} t+\lambda N \wedge t,\langle d N(t), a\rangle=0$ and $\langle N \wedge t, a\rangle \neq 0$ we conclude $\lambda=0$ and $\Gamma$ is a line of curvature. The result follows from Lemma 2.

By combining Theorem 2 and an isoperimetric inequality due to Barbosa and do Carmo (see [1]), we have

Corollary 2. Let $\Gamma$ be a planar Jordan curve. Then

$$
2 \pi-2 \sqrt{\pi^{2}-\pi K|\bar{A}|} \leq K A \leq 2 \pi+2 \sqrt{\pi^{2}-\pi K|\bar{A}|} .
$$

If the length $L$ of $\Gamma$ satisfies $4 \pi^{2}-K L^{2} \geq 0$, we have either

$$
2 \pi-2 \sqrt{\pi^{2}-\pi K|\bar{A}|} \leq K A \leq 2 \pi-\sqrt{4 \pi^{2}-K L^{2}}
$$

or

$$
2 \pi+\sqrt{4 \pi^{2}-K L^{2}} \leq K A \leq 2 \pi+2 \sqrt{\pi^{2}-\pi K|\bar{A}|} .
$$

Moreover, the equality holds in some of the above inequalities if and only if $x(S)$ is a spherical cap.

Proof of Theorem 3. Let us assume $x(u, v)=(u, v, f(u, v)),(u, v) \in \Omega \subseteq P$. If $\Gamma_{s}$ denotes the planar Jordan curve given by $x(\Omega) \cap\{(u, v, s) \mid u, v \in \mathbb{R}\}$, then from (4) and (6)

$$
\begin{aligned}
2 K V & =2 K \int_{0}^{h}\left|\bar{A}\left(\Gamma_{s}\right)\right|=\int_{0}^{h} \int_{\Gamma_{s}}\langle d N \wedge N, a\rangle \\
& =\int_{0}^{h} \int_{\Gamma_{s}} k_{n}\left(\Gamma_{s}\right)\left\langle N \wedge t^{s}, a\right\rangle=\int_{0}^{h} \int_{\Gamma_{s}} k_{\Gamma_{s}}|\nabla f|^{2},
\end{aligned}
$$

where $t^{s}$ is a well-oriented unit tangent vector along $\Gamma_{s}, k_{n}\left(\Gamma_{s}\right)$ and $k_{\Gamma_{s}}$ are the normal curvature and the Euclidean curvature of $\Gamma_{s}$, respectively, and $f=\langle x, a\rangle$ is the height function.

From inequality (2), $\sqrt{K} f+\langle N, a\rangle \leq 0$ on $\Omega$. Then, by using that $\langle N, a\rangle=$ $-1 / \sqrt{1+f_{u}^{2}+f_{v}^{2}}$, we have

$$
f_{u}^{2}+f_{v}^{2}=\frac{1}{\langle N, a\rangle^{2}}-1 \leq \frac{1}{K f^{2}}-1
$$

and

$$
|\nabla f|^{2} \leq 1-K f^{2} .
$$


From (9) we obtain

$$
2 K V \leq \int_{0}^{h}\left(1-K s^{2}\right) \int_{\Gamma_{s}} k_{\Gamma_{s}}=2 \pi\left(h-\frac{K h^{3}}{3}\right),
$$

which gives the inequality in the theorem.

Moreover the equality holds if and only if $\Gamma_{s}$ is a line of curvature for all $s$. Now, the result follows from Lemma 2.

As a consequence of the above theorem and (2) we have

Corollary 3. If $x$ is a graph on a domain $\Omega \subseteq P$, then

$$
V \leq \frac{2}{3} \pi\left(\frac{1}{\sqrt{K}}\right)^{3}
$$

and the equality holds if and only if $x(\Omega)$ is a hemisphere.

\section{REFERENCES}

[1] J. L. BARbosa and M. P. Do CARmo, 'A proof of a general isoperimetric inequality for surfaces', Math. Z., 162 (1978), 245-261. MR 80f:53043

[2] L. A. Caffarelli, L. Nirenberg and J. Spruck, 'The Dirichlet problem for nonlinear second-order elliptic equations I. Monge-Ampère equations', Comm. Pure Appl. Math., 37 (1984), 369-402. MR 87f:35096 corrections MR 88k:35073

[3] J. A. GÁLvez and A. Martínez, 'The Gauss map and second fundamental form of surfaces in $\mathbb{R}^{3}$, Geom. Dedicata (to appear).

[4] B. Guan and J. Spruck, 'Boundary value problems on $\mathbb{S}^{n}$ for surfaces of constant Gauss curvature', Ann. Math., 138 (1993), 601-624. MR 94i:53039

[5] D. Hoffman, H. Rosenberg and J. Spruck, 'Boundary value problems for surfaces of constant Gauss curvature', Comm. Pure Appl. Math., vol. XLV, (1992), 1051-1062. MR 93h:53009

[6] H. Rosenberg, 'Hypersurfaces of constant curvature in space forms', Bull. Sc. math., $2^{e}$ série, 117 (1993), 211-239. MR 94b:53097

Departamento de Geometría y Topología, Facultad de Ciencias, Universidad de Granada, 18071 Granada, Spain

E-mail address: jagalvez@goliat.ugr.es

Departamento de Geometría y Topología, Facultad de Ciencias, Universidad de Granada, 18071 Granada, Spain

E-mail address: amartine@goliat.ugr.es 